\title{
Inviscid Quasi-Neutral Limit of a Navier-Stokes-Poisson-Korteweg System
}

\section{Hongli Wang ${ }^{a}$ and Jianwei Yang ${ }^{a}$}

\author{
${ }^{a}$ School of Mathematics and Statistics, North China University of Water \\ Resources and Electric Power \\ 450045 Zhengzhou, Henan Province, China \\ E-mail(corresp.): yangjianwei@ncwu.edu.cn \\ E-mail: wanghongli@ncwu.edu.cn
}

Received August 8, 2017; revised January 21, 2018; accepted January 22, 2018

\begin{abstract}
The combined quasi-neutral and inviscid limit of the Navier-StokesPoisson-Korteweg system with density-dependent viscosity and cold pressure in the torus $\mathbb{T}^{3}$ is studied. It is shown that, for the well-prepared initial data, the global weak solution of the Navier-Stokes-Poisson-Korteweg system converges strongly to the strong solution of the incompressible Euler equations when the Debye length and the viscosity coefficient go to zero simultaneously. Furthermore, the rate of convergence is also obtained.
\end{abstract}

Keywords: incompressible Euler equations, inviscid limit, Navier-Stokes-Poisson-Korteweg system, quasi-neutral limit.

AMS Subject Classification: 35Q35; 35B40; 76N10.

\section{Introduction and main results}

Hydrodynamical models have been widely used to describe the physical phenomena in plasmas and semiconductors physics [21]. In the inviscid case, the Euler-Poisson system has been extensively studied. In the viscid case, where the viscous stress tensors are taken into consideration, the most common model is the compressible Navier-Stokes-Poisson system. Moreover, in the particular case, it is necessary to add to the momentum equation a capillarity tensor if we take under consideration the surface tension effects. This type of models was first introduced by Korteweg [16], and derived rigorously by Dunn and Serrin [10]. In this paper, we are concerned with the rigorous asymptotic analysis

Copyright (c) 2018 The Author(s). Published by VGTU Press

This is an Open Access article distributed under the terms of the Creative Commons Attribution License (http://creativecommons.org/licenses/by/4.0/), which permits unrestricted use, distribution, and reproduction in any medium, provided the original author and source are credited. 
of the following scaled Navier-Stokes-Poisson-Korteweg (NSPK) system

$$
\begin{aligned}
& \partial_{t} n+\operatorname{div}(n u)=0, \\
& \partial_{t}(n u)+\operatorname{div}(n u \otimes u)+\nabla\left(p(n)+p_{c}(n)\right) \\
& =-n \nabla \Phi+2 \mu \operatorname{div}(n D(u))+\kappa n \nabla \Delta n, \\
& -\lambda^{2} \Delta \Phi=n-1
\end{aligned}
$$

with initial data

$$
n(x, 0)=n_{0}, \quad u(x, 0)=u_{0}, \quad \Phi(x, 0)=\Phi_{0}
$$

satisfying compatibility condition

$$
-\lambda^{2} \Delta \Phi_{0}=n_{0}-1
$$

for $x \in \mathbb{T}^{3}, t>0$. Here, $\mathbb{T}^{3}$ is the torus in $\mathbb{R}^{3} . \mu>0$ denotes viscosity coefficient, $\kappa>0$ is the Weber number and $\lambda>0$ is the Debye length. The unknown functions are the density $n$, the velocity $u$ and the electrostatic potential $\Phi$, respectively. In this paper, we suppose that $p(n)=a^{2} n^{\gamma}$ with constants $\gamma>1$ and $a \neq 0$. The cold pressure $p_{c}(n)$ is a singular continuous function namely a suitable increasing function as introduced in [3]. More precisely, we assume that $p_{c}(n)=-\frac{3 a^{2} \epsilon}{2(\gamma-1) n^{2}}$ for some constant $\epsilon>0 . D(u)=\frac{1}{2}\left(\nabla u+\nabla u^{T}\right)$ is the symmetric part of the velocity gradient.

Recently, Li-Yong [20] proved that the quasi-neutral limit $\lambda \rightarrow 0$ of the NSPK system (1.1)-(1.3) with common viscosity and without cold pressure is the following incompressible Navies-Stokes equations:

$$
\partial_{t} u^{0}+\left(u^{0} \cdot \nabla\right) u^{0}+\nabla p^{0}=\mu \Delta u^{0}, \quad \operatorname{div} u^{0}=0
$$

for (local) smooth solution in the two or three dimensional torus.

Our objective of this paper is to justify rigorously the convergence of the NSPK system (1.1)-(1.3) to the following ideal incompressible Euler equations

$$
\partial_{t} u^{0}+\left(u^{0} \cdot \nabla\right) u^{0}+\nabla p^{0}=0, \quad \operatorname{div} u^{0}=0
$$

for global weak solutions by performing the combined quasi-neutral and vanishing viscosity limit $\lambda \rightarrow 0$ and $\mu \rightarrow 0$ in the torus $\mathbb{T}^{3}$. In this paper, we assume that $\mu=O(\lambda)$. Let $\Psi^{\lambda}=\lambda \Phi$ and rewrite the NSPK system (1.1)-(1.3) into the following form

$$
\begin{aligned}
& \partial_{t} n^{\lambda}+\operatorname{div}\left(n^{\lambda} u^{\lambda}\right)=0 \\
& \partial_{t}\left(n^{\lambda} u^{\lambda}\right)+\operatorname{div}\left(n^{\lambda} u^{\lambda} \otimes u^{\lambda}\right)+\nabla\left(p\left(n^{\lambda}\right)+p_{c}\left(n^{\lambda}\right)\right) \\
& =-\frac{n^{\lambda} \nabla \Psi^{\lambda}}{\lambda}+2 \mu \operatorname{div}\left(n^{\lambda} D\left(u^{\lambda}\right)\right)+\kappa n^{\lambda} \nabla \Delta n^{\lambda}, \\
& -\lambda \Delta \Psi^{\lambda}=n^{\lambda}-1
\end{aligned}
$$

with initial data

$$
n^{\lambda}(x, 0)=n_{0}^{\lambda}, \quad u^{\lambda}(x, 0)=u_{0}^{\lambda}
$$


Before stating our main result, we first recall the local existence of strong solution to the incompressible Euler equations (1.4) and the global existence of weak solution to the NSPK system (1.5)-(1.7).

Proposition 1. (Ref. [15]) Let $s>\frac{5}{2}+1$ be an integer. Assume that the initial data $u^{0}(x, 0)=u_{0}^{0}(x)$ satisfies $u_{0}^{0} \in H^{s}\left(\mathbb{T}^{3}\right)$ and divu $u_{0}^{0}=0$. Then there exists $a T_{*}>0$ and a unique solution $u^{0} \in L^{\infty}\left([0, T] ; H^{s}\left(\mathbb{T}^{3}\right)\right)$ to the incompressible Euler equations (1.4) with the initial data $u_{0}^{0}(x)$ satisfying for any $T \in\left(0, T_{*}\right)$

$$
\sup _{t \in[0, T]}\left(\left\|u^{0}, \nabla p^{0}\right\|_{s}+\left\|\partial_{t} u^{0}, \partial_{t} \nabla p^{0}\right\|_{s-1}\right) \leq C_{T}
$$

for some positive constant $C_{T}$, depending only on $T$.

Yang-Wang-Ding [30] prove the existence of global weak solutions to system (1.1)-(1.3) by using the Faedo-Galerkin approximation method the compactness argument.

Proposition 2. ([30]) Let $T>0$. Assume that the initial data $\left(n_{0}^{\lambda}, u_{0}^{\lambda}, \Psi_{0}^{\lambda}\right)$ satisfy (1.8) and the compatibility condition $\int_{\mathbb{T}^{3}}\left(n_{0}^{\lambda}-1\right) d x=0$, where $\Psi_{0}^{\lambda}$ is given by $-\lambda \Delta \Psi_{0}^{\lambda}=n_{0}^{\lambda}-1$. Then there exists a weak solution $\left(n^{\lambda}, u^{\lambda}, \Psi^{\lambda}\right)$ of (1.5) $-(1.7)$.

1. $n^{\lambda} \in L^{\infty}\left([0, T] ; L^{\gamma}\left(\mathbb{T}^{3}\right)\right), \quad \nabla n^{\lambda} \in L^{\infty}\left([0, T] ; L^{2}\left(\mathbb{T}^{3}\right)\right)$,

$\frac{1}{n^{\lambda}} \in L^{\infty}\left([0, T] ; L^{2}\left(\mathbb{T}^{3}\right)\right), \quad \nabla\left(\frac{1}{n^{\lambda}}\right) \in L^{\infty}\left([0, T] ; L^{2}\left(\mathbb{T}^{3}\right)\right)$,

$\sqrt{n^{\lambda}} u^{\lambda} \in L^{\infty}\left([0, T] ; L^{2}\left(\mathbb{T}^{3}\right)\right), \sqrt{n^{\lambda}}\left|\nabla u^{\lambda}\right| \in L^{2}\left([0, T] ; L^{2}\left(\mathbb{T}^{3}\right)\right)$,

$\nabla \Psi^{\lambda} \in L^{\infty}\left([0, T] ; L^{2}\left(\mathbb{T}^{3}\right)\right), \Delta \Psi^{\lambda} \in L^{2}\left([0, T] ; L^{2}\left(\mathbb{T}^{3}\right)\right)$.

2. The energy inequality

$$
E^{\lambda}(t)+2 \mu \int_{0}^{t} \int_{\mathbb{T}^{3}} n^{\lambda}\left|D\left(u^{\lambda}\right)\right|^{2} d x d \tau \leq E^{\lambda}(0)
$$

holds with the finite total energy

$$
E^{\lambda}(t)=\frac{1}{2} \int_{\mathbb{T}^{3}}\left(n^{\lambda}\left|u^{\lambda}\right|^{2}+h\left(n^{\lambda}\right)+\kappa\left|\nabla n^{\lambda}\right|^{2}+\left|\nabla \Psi^{\lambda}\right|^{2}\right) d x,
$$

where

$$
h\left(n^{\lambda}\right)=\frac{2 a^{2}}{\gamma-1}\left[\left(n^{\lambda}\right)^{\gamma}-1-(\gamma-\epsilon)\left(n^{\lambda}-1\right)+\frac{\epsilon}{2}\left(\frac{1}{\left(n^{\lambda}\right)^{2}}-1\right)\right]
$$

is a convex on $[0,+\infty)$ since $\gamma>1$.

3. The system $(1.5)-(1.7)$ holds in $\mathcal{D}^{\prime}\left(\mathbb{T}^{3} \times(0,+\infty)\right)$.

Throughout this article, we use $C$ to denote the positive constant independent of $\lambda$ and $\mu$, which can be different from line to another line. The main result of this paper reads as follows. 
Theorem 1. Assume that the initial data $\left(n_{0}^{\lambda}, u_{0}^{\lambda}, \Psi_{0}^{\lambda}\right)$ satisfy the assumptions in Proposition 2. Assume further that they satisfy

$$
\int_{\mathbb{T}^{3}} h\left(n_{0}^{\lambda}\right) d x+\left\|\nabla n_{0}^{\lambda}\right\|_{L^{2}\left(\mathbb{T}^{3}\right)}^{2}+\left\|\sqrt{n_{0}^{\lambda}} u_{0}^{\lambda}-u_{0}^{0}\right\|_{L^{2}\left(\mathbb{T}^{3}\right)}^{2}+\left\|\nabla \Psi_{0}^{\lambda}\right\|_{L^{2}\left(\mathbb{T}^{3}\right)}^{2} \leq C \lambda .
$$

Let $u^{0}$ be the smooth solution, defined on $\left[0, T_{*}\right)$, to the incompressible Euler equations (1.4) with initial data $u_{0}^{0}$. Then for any $0<T<T_{*}$, the global weak solution $\left(n^{\lambda}, u^{\lambda}, \Psi^{\lambda}\right)$ of the NSPK system (1.5)-(1.7) satisfies the following estimates

$$
\begin{aligned}
\left\|n^{\lambda}-1\right\|_{L^{\infty}\left([0, T] ; L^{\gamma}\left(\mathbb{T}^{3}\right)\right)}^{\gamma} & \leq C \lambda^{\min \left\{\frac{\gamma^{2}}{4}, 1\right\}}, \\
\left\|\sqrt{n^{\lambda}} u^{\lambda}-u^{0}\right\|_{L^{\infty}\left([0, T] ; L^{2}\left(\mathbb{T}^{3}\right)\right)}^{2} & \leq C \lambda^{\min \left\{\frac{\gamma^{2}}{4}, 1\right\}}, \\
\left\|\nabla n^{\lambda}\right\|_{L^{\infty}\left([0, T] ; L^{2}\left(\mathbb{T}^{3}\right)\right)}^{2} & \leq C \lambda^{\min \left\{\frac{\gamma}{2}, 1\right\}}, \\
\left\|\nabla \Psi^{\lambda}\right\|_{L^{\infty}\left([0, T] ; L^{2}\left(\mathbb{T}^{3}\right)\right)}^{2} & \leq C \lambda^{\min \left\{\frac{\gamma}{2}, 1\right\}}, \\
\left\|n^{\lambda} u^{\lambda}-u^{0}\right\|_{L^{\infty}([0, T] ; L}^{2} & \leq C \lambda^{\min \left\{\frac{\gamma^{2}}{4}, 1\right\}} .
\end{aligned}
$$

Remark 1. Theorem 1 describes the combined quasi-neutral and vanishing viscosity limit of the NSPK system (1.5)-(1.7) with well-prepared initial data. For the general initial data, there are oscillations in time of the solution sequence because the oscillation part with respect to time $\frac{t}{\lambda}$ does not vanish. It is more difficult to prove the asymptotic limit in this situation, which will be studied in our future work.

The proof of Theorem 1 is based on the modulated energy method, first introduced by Brenier in a kinetic context [2] and later extended to various models, e.g. $[1,14,18,27]$. The idea of modulated energy method is to modulate the energy of the given system by test functions, and to obtain a stability inequality when these test functions are the solution to the limiting system. Noticing that our result is different from that in Ref. [20], where the convergence of (local) smooth solution of the NSPK system with viscosity term $\mu \Delta u^{\lambda}+\nu \nabla \operatorname{div} u^{\lambda}$ to the smooth solution of the incompressible Navier-Stokes equations is obtained by using the convergence-stability principle. It is also different from that in Ref. [4], where the limit system is the compressible capillary Navier-Stokes equations. In this paper, we consider the convergence of global weak solution of the NSPK system with density-dependent viscosity term $\mu \operatorname{div}\left(n D\left(u^{\lambda}\right)\right)$ and cold pressure term $p_{c}\left(n^{\lambda}\right)$ to the smooth solution of the incompressible Euler equations by using the relative entropy theory.

Before ending this introduction, let us mention that the quasi-neutral limit of fluid dynamic models and of kinetic models of semiconductors and plasmas has attracted much attention. In particular, the quasi-neutral limit $\lambda \rightarrow 0$ has been studied in Vlasov-Poisson system by Brenier [2], Grenier [12] and Masmoudi [22], in drift-diffusion model by Gasser et al. [11], Wang et al. [28], in Euler-Poisson system by Cordier-Grenier [5], Wang [26], Peng et al. [24] and Slemrod-Sternberg [25], in Navier-Stokes-Poisson system by Wang-Jiang 
[27], Ju et al. [13], Donatelli et al. [6], Donatelli-Marcati [7], in Navier-StokesFourier-Poisson system by Donatelli-Marcati [8], in Euler-Maxwell system [23], in electro-diffusion system by $\mathrm{Li}$ [17], in quantum hydrodynamic model by Li [18], Li-Lin [19] and Yang-Ju [29], in Korteweg type fluids by DonatelliMarcati [9].

The remainder of this paper is devoted to proving Theorem 1.

\section{Proof of Theorem 1}

We define the modulated energy functional $\mathcal{H}^{\lambda}(t)$ by

$$
\mathcal{H}^{\lambda}(t)=\frac{1}{2} \int_{\mathbb{T}^{3}}\left\{n^{\lambda}\left|u^{\lambda}-u^{0}\right|^{2}+h\left(n^{\lambda}\right)+\kappa\left|\nabla n^{\lambda}\right|^{2}+\left|\nabla \Psi^{\lambda}\right|^{2}\right\} d x,
$$

where $u^{0}$ is the smooth solution of the incompressible Euler equations (1.4). These terms express the differences of the kinetic energy, internal energy, Korteweg energy and electric field energy. Using the careful energy method, we are able to prove that

$$
\mathcal{H}^{\lambda}(t) \leq C \int_{0}^{t} \mathcal{H}^{\lambda}(\tau) d \tau+C \lambda^{\theta}
$$

with some positive constant $\theta$. The Gronwall lemma then implies $\mathcal{H}^{\lambda}(t) \rightarrow 0$ as $\lambda \rightarrow 0$, which yields the desired convergence results.

To derive the integration inequality for $\mathcal{H}^{\lambda}(t)$, we use $u^{0}$ as a test function in the weak formulation of momentum equation (1.6) to yield the following equality for almost all $t$.

$$
\begin{aligned}
& \int_{\mathbb{T}^{3}} n^{\lambda} u^{\lambda} \cdot u^{0} d x=\int_{\mathbb{T}^{3}} n_{0}^{\lambda} u_{0}^{\lambda} \cdot u_{0}^{0} d x+\int_{0}^{t} \int_{\mathbb{T}^{3}} n^{\lambda} u^{\lambda} \cdot \partial_{\tau} u^{0} d x d \tau \\
& +\int_{0}^{t} \int_{\mathbb{T}^{3}}\left(n^{\lambda} u^{\lambda} \otimes u^{\lambda}\right): \nabla u^{0} d x d \tau+\kappa \int_{0}^{t} \int_{\mathbb{T}^{3}}\left(\nabla n^{\lambda} \otimes \nabla n^{\lambda}\right): \nabla u^{0} d x d \tau \\
& -2 \mu \int_{0}^{t} \int_{\mathbb{T}^{3}} n^{\lambda} D\left(u^{\lambda}\right): D\left(u^{0}\right) d x d \tau-\int_{0}^{t} \int_{\mathbb{T}^{3}}\left(\nabla \Psi^{\lambda} \times \nabla \Psi^{\lambda}\right): \nabla u^{0} d x d \tau,
\end{aligned}
$$

where we have used $\operatorname{div} u^{0}=0$ and the following relations

$$
\begin{aligned}
n^{\lambda} \nabla \Delta n^{\lambda} & =\frac{1}{2} \nabla \Delta\left(n^{\lambda}\right)^{2}-\frac{1}{2} \nabla\left|\nabla n^{\lambda}\right|^{2}-\operatorname{div}\left(\nabla n^{\lambda} \otimes \nabla n^{\lambda}\right), \\
-\frac{n \nabla \Psi^{\lambda}}{\lambda} & =-\frac{\nabla \Psi^{\lambda}}{\lambda}+\Delta \Psi^{\lambda} \nabla \Psi^{\lambda}=-\frac{\nabla \Psi^{\lambda}}{\lambda}+\operatorname{div}\left(\nabla \Psi^{\lambda} \otimes \nabla \Psi^{\lambda}\right)-\frac{1}{2} \nabla\left|\nabla \Psi^{\lambda}\right|^{2} .
\end{aligned}
$$

Multiplying (1.4) by $u^{0}$ and using the property

$$
\int_{0}^{t} \int_{\mathbb{T}^{3}}\left(u^{0} \cdot \nabla\right) u^{0} \cdot u^{0} d x=\frac{1}{2} \int_{0}^{t} \int_{\mathbb{T}^{3}} u^{0} \cdot \nabla\left|u^{0}\right|^{2} d x d \tau=0
$$

we find that the energy identity of the incompressible Euler equations reads

$$
\frac{1}{2} \frac{d}{d t} \int_{\mathbb{T}^{3}}\left|u^{0}\right|^{2} d x=0
$$


which implies that

$$
\frac{1}{2} \int_{\mathbb{T}^{3}}\left|u^{0}\right|^{2} d x=\frac{1}{2} \int_{\mathbb{T}^{3}}\left|u_{0}^{0}\right|^{2} d x .
$$

Using (2.1), (2.3) and the energy inequality (1.10) by integration by parts we can calculate $\mathcal{H}^{\lambda}(t)$ as follows.

$$
\begin{aligned}
\mathcal{H}^{\lambda}( & t)+2 \mu \int_{0}^{t} \int_{\mathbb{T}^{3}} n^{\lambda}\left|D\left(u^{\lambda}\right)\right|^{2} d x d \tau=E^{\lambda}(t)+2 \mu \int_{0}^{t} \int_{\mathbb{T}^{3}} n^{\lambda}\left|D\left(u^{\lambda}\right)\right|^{2} d x d \tau \\
& +\frac{1}{2} \int_{\mathbb{T}^{3}}\left|u^{0}\right|^{2} d x+\frac{1}{2} \int_{\mathbb{T}^{3}}\left(n^{\lambda}-1\right)\left|u^{0}\right|^{2} d x-\int_{\mathbb{T}^{3}} n^{\lambda} u^{\lambda} \cdot u^{0} d x \\
\leq & E^{\lambda}(0)+\frac{1}{2} \int_{\mathbb{T}^{3}}\left|u_{0}^{0}\right|^{2} d x-\int_{\mathbb{T}^{3}} n_{0}^{\lambda} u_{0}^{\lambda} \cdot u_{0}^{0} d x+\frac{1}{2} \int_{\mathbb{T}^{3}}\left(n^{\lambda}-1\right)\left|u^{0}\right|^{2} d x \\
& -\int_{0}^{t} \int_{\mathbb{T}^{3}} n^{\lambda} u^{\lambda} \cdot \partial_{\tau} u^{0} d x d \tau-\int_{0}^{t} \int_{\mathbb{T}^{3}}\left(n^{\lambda} u^{\lambda} \otimes u^{\lambda}\right): \nabla u^{0} d x d \tau \\
& -\kappa \int_{0}^{t} \int_{\mathbb{T}^{3}}\left(\nabla n^{\lambda} \otimes \nabla n^{\lambda}\right): \nabla u^{0} d x d \tau+\int_{0}^{t} \int_{\mathbb{T}^{3}}\left(\nabla \Psi^{\lambda} \otimes \nabla \Psi^{\lambda}\right): \nabla u^{0} d x d \tau \\
& +2 \mu \int_{0}^{t} \int_{\mathbb{T}^{3}} n^{\lambda} D\left(u^{\lambda}\right): D\left(u^{0}\right) d x d \tau=\mathcal{H}^{\lambda}(0)-\frac{1}{2} \int_{\mathbb{T}^{3}}\left(n_{0}^{\lambda}-1\right)\left|u_{0}^{0}\right|^{2} d x \\
& +\frac{1}{2} \int_{\mathbb{T}^{3}}\left(n^{\lambda}-1\right)\left|u^{0}\right|^{2} d x-\int_{0}^{t} \int_{\mathbb{T}^{3}} n^{\lambda} u^{\lambda} \cdot \partial_{\tau} u^{0} d x d \tau \\
- & \int_{0}^{t} \int_{\mathbb{T}^{3}}\left(n^{\lambda} u^{\lambda} \otimes u^{\lambda}\right): \nabla u^{0} d x d \tau-\kappa \int_{0}^{t} \int_{\mathbb{T}^{3}}\left(\nabla n^{\lambda} \otimes \nabla n^{\lambda}\right): \nabla u^{0} d x d \tau \\
+ & \int_{0}^{t} \int_{\mathbb{T}^{3}}\left(\nabla \Psi^{\lambda} \otimes \nabla \Psi^{\lambda}\right): \nabla u^{0} d x d \tau+2 \mu \int_{0}^{t} \int_{\mathbb{T}^{3}} n^{\lambda} D\left(u^{\lambda}\right): D\left(u^{0}\right) d x d \tau \\
= & \mathbf{I}_{1}+\mathbf{I}_{2}+\mathbf{I}_{3}+\mathbf{I}_{4}+\mathbf{I}_{5}+\mathbf{I}_{6}+\mathbf{I}_{7}+\mathbf{I}_{8} .
\end{aligned}
$$

Next, we begin to treat the nine integrals $\mathbf{I}_{k}(k=1,2, \ldots, 8)$ term by term. To estimate the term $\mathbf{I}_{1}$, we need to prove the following lemma.

Lemma 1. Assume that $n_{0}^{\lambda}$ satisfies (1.11). Then there exists a constant $C>0$ such that for $\forall \lambda \in(0,1)$

$$
\left\|n_{0}^{\lambda}-1\right\|_{L^{\infty}\left([0, T] ; L^{\gamma}\left(\mathbb{T}^{3}\right)\right)} \leq C \lambda^{\min \left\{\frac{1}{2}, \frac{1}{\gamma}\right\}} .
$$

Proof. We can deduce the following basic fact: for all $x \geq 0$ and for $\forall \mathcal{R} \in$ $(1,+\infty)$

$$
\begin{aligned}
& x^{\gamma}-1-(\gamma-\epsilon)(x-1)+\frac{\epsilon}{2}\left(\frac{1}{x^{2}}-1\right) \geq c_{1}|x-1|^{\gamma} \quad \text { if } \gamma \geq 2, \\
& x^{\gamma}-1-(\gamma-\epsilon)(x-1)+\frac{\epsilon}{2}\left(\frac{1}{x^{2}}-1\right) \geq c_{2}|x-1|^{2} \quad \text { if } \gamma<2, x \leq \mathcal{R}, \\
& x^{\gamma}-1-(\gamma-\epsilon)(x-1)+\frac{\epsilon}{2}\left(\frac{1}{x^{2}}-1\right) \geq c_{3}|x-1|^{\gamma} \text { if } \gamma<2, x>\mathcal{R},
\end{aligned}
$$


where,

$$
c_{1}=\min \left\{1, \frac{3 \epsilon}{\gamma(\gamma-1)}\right\}, \quad c_{2}=\frac{3 \epsilon}{2 \mathcal{R}^{4}}, \quad c_{3}=\left(\frac{\mathcal{R}}{\mathcal{R}-1}\right)^{\gamma-2} .
$$

Hence, for $\gamma \geq 2$, we have

$$
\left\|n_{0}^{\lambda}-1\right\|_{L^{\gamma}\left(\mathbb{T}^{3}\right)}^{\gamma} \leq C \int_{\mathbb{T}^{3}} h\left(n_{0}^{\lambda}\right) d x \leq C \lambda
$$

For $\gamma<2$, using Höder's inequality, we have

$$
\begin{aligned}
& \left\|n_{0}^{\lambda}-1\right\|_{L^{\gamma}\left(\mathbb{T}^{3}\right)}^{\gamma} \leq C\left(\int_{\left\{n_{0}^{\lambda} \leq \mathcal{R}\right\}}\left|n_{0}^{\lambda}-1\right|^{2} d x\right)^{\frac{\gamma}{2}}+\int_{\left\{n_{0}^{\lambda}>\mathcal{R}\right\}}\left|n_{0}^{\lambda}-1\right|^{\gamma} d x \\
& \quad \leq C\left(\int_{\left\{n_{0}^{\lambda} \leq \mathcal{R}\right\}} h\left(n_{0}^{\lambda}\right) d x\right)^{\frac{\gamma}{2}}+C \int_{\left\{n_{0}^{\lambda}>\mathcal{R}\right\}} h\left(n_{0}^{\lambda}\right) d x \leq C\left(\lambda^{\frac{\gamma}{2}}+\lambda\right) \leq C \lambda^{\frac{\gamma}{2}} .
\end{aligned}
$$

The inequalities (2.5) and (2.6) finish the proof.

Using Lemma 1, we have

$$
\begin{gathered}
\int_{\mathbb{T}^{3}} n_{0}^{\lambda}\left|u_{0}^{\lambda}-u_{0}^{0}\right|^{2} d x \leq 2 \int_{\mathbb{T}^{3}}\left|\sqrt{n_{0}^{\lambda}} u_{0}^{\lambda}-u_{0}^{0}\right|^{2} d x+2 \int_{\mathbb{T}^{3}}\left|\left(1-\sqrt{n_{0}^{\lambda}}\right) u_{0}^{0}\right|^{2} d x \\
\leq 2 \int_{\mathbb{T}^{3}}\left|\sqrt{n_{0}^{\lambda}} u_{0}^{\lambda}-u_{0}^{0}\right|^{2} d x+C \int_{\mathbb{T}^{3}}\left|1-\sqrt{n_{0}^{\lambda}}\right|^{2} d x \\
\leq 2 \int_{\mathbb{T}^{3}}\left|\sqrt{n_{0}^{\lambda}} u_{0}^{\lambda}-u_{0}^{0}\right|^{2} d x+C \int_{\mathbb{T}^{3}}\left|1-n_{0}^{\lambda}\right|^{\gamma} d x \leq C \lambda^{\min \left\{\frac{\gamma}{2}, 1\right\}},
\end{gathered}
$$

where we have used the following elementary inequality

$$
|1-\sqrt{x}|^{2} \leq C|1-x|^{\gamma}, \quad \gamma>1
$$

for some positive constant $C$ and $\forall x \geq 0$. Then, from (1.11), we get

$$
\mathbf{I}_{1}=\mathcal{H}^{\lambda}(0) \leq C \lambda^{\min \left\{\frac{\gamma}{2}, 1\right\}} .
$$

The integrals $\mathbf{I}_{2}$ and $\mathbf{I}_{3}$ cancel with a contribution originating from $\mathbf{I}_{5}$ (see below). Using (1.4), we have

$$
\begin{aligned}
\mathbf{I}_{4} & =\int_{0}^{t} \int_{\mathbb{T}^{3}} n^{\lambda} u^{\lambda} \cdot\left(\left(u^{0} \cdot \nabla\right) u^{0}+\nabla p^{0}\right) d x d \tau \\
& =\int_{0}^{t} \int_{\mathbb{T}^{3}} n^{\lambda} u^{\lambda} \cdot\left(\left(u^{0} \cdot \nabla\right) u^{0}\right) d x d \tau+\int_{0}^{t} \int_{\mathbb{T}^{3}} n^{\lambda} u^{\lambda} \cdot \nabla p^{0} d x d \tau .
\end{aligned}
$$

In fact, we only need to treat the second term on the right hand side of the above inequality and the first term will be canceled later (See (2.10)). Using 
the $L^{\infty}\left([0, T] ; L^{2}\left(\mathbb{T}^{3}\right)\right)$ bound on $\nabla \Psi^{\lambda}$ due to the energy inequality (1.10), the continuity equation (1.5) and Poisson equation (1.7), we get

$$
\begin{aligned}
& \int_{0}^{t} \int_{\mathbb{T}^{3}} n^{\lambda} u^{\lambda} \cdot \nabla p^{0} d x d \tau=-\lambda \int_{0}^{t} \int_{\mathbb{T}^{3}} \Delta\left(\partial_{\tau} \Psi^{\lambda}\right) p^{0} d x d \tau \\
& =\lambda \int_{0}^{t} \int_{\mathbb{T}^{3}} \nabla\left(\partial_{\tau} \Psi^{\lambda}\right) \cdot p^{0} d x d \tau=\lambda \int_{\mathbb{T}^{3}} \nabla \Psi^{\lambda} \cdot p^{0} d x-\lambda \int_{\mathbb{T}^{3}} \nabla \psi_{0}^{\lambda} \cdot p_{0}^{0} d x \\
& \quad-\lambda \int_{0}^{t} \int_{\mathbb{T}^{3}} \nabla \Psi^{\lambda} \cdot \partial_{\tau} \nabla p^{0} d x d \tau \leq C \lambda .
\end{aligned}
$$

Therefore, we have that

$$
\mathbf{I}_{4} \leq \int_{0}^{t} \int_{\mathbb{T}^{3}} n^{\lambda} u^{\lambda} \cdot\left(\left(u^{0} \cdot \nabla\right) u^{0}\right) d x d \tau+C \lambda .
$$

In order to treat the term $\mathbf{I}_{5}$, we add and subtract the expression $u^{0}$ such that

$$
\begin{aligned}
\mathbf{I}_{5}= & -\int_{0}^{t} \int_{\mathbb{T}^{3}} n^{\lambda}\left(u^{\lambda}-u^{0}\right) \otimes\left(u^{\lambda}-u\right): \nabla u^{0} d x d \tau-\int_{0}^{t} \int_{\mathbb{T}^{3}}\left(n^{\lambda} u^{0} \otimes u^{\lambda}\right): \nabla u^{0} d x d \tau \\
& +\int_{0}^{t} \int_{\mathbb{T}^{3}}\left(n^{\lambda} u^{0} \otimes u^{0}\right): \nabla u^{0} d x d \tau-\int_{0}^{t} \int_{\mathbb{T}^{3}}\left(n^{\lambda} u^{\lambda} \otimes u^{0}\right): \nabla u^{0} d x d \tau \\
\leq & C \int_{0}^{t} \mathcal{H}^{\lambda}(\tau) d \tau-\int_{0}^{t} \int_{\mathbb{T}^{3}} n^{\lambda} u^{\lambda} \cdot\left(\left(u^{0} \cdot \nabla\right) u^{0}\right) d x d \tau+\mathbf{I}_{51}+\mathbf{I}_{52},
\end{aligned}
$$

where,

$$
\mathbf{I}_{51}=\int_{0}^{t} \int_{\mathbb{T}^{3}}\left(n^{\lambda} u^{0} \otimes u^{0}\right): \nabla u^{0} d x d \tau, \quad \mathbf{I}_{52}=-\int_{0}^{t} \int_{\mathbb{T}^{3}}\left(n^{\lambda} u^{\lambda} \otimes u^{0}\right): \nabla u^{0} d x d \tau .
$$

Using the uniform bound on $\nabla \Psi^{\lambda}$ due to the energy inequality (1.10), the Poisson equation (1.7), the equality (2.2)and integration by parts, we get

$$
\begin{aligned}
\mathbf{I}_{51} & =\int_{0}^{t} \int_{\mathbb{T}^{3}} n^{\lambda}\left(u^{0} \cdot \nabla\right) u^{0} \cdot u^{0} d x d \tau=\int_{0}^{t} \int_{\mathbb{T}^{3}}\left(n^{\lambda}-1\right)\left(u^{0} \cdot \nabla\right) u^{0} \cdot u^{0} d x d \tau \\
& =\lambda \int_{0}^{t} \int_{\mathbb{T}^{3}} \nabla \Psi^{\lambda} \cdot \nabla\left(\left(u^{0} \cdot \nabla\right) u^{0} \cdot u^{0}\right) d x d \tau \leq C \lambda .
\end{aligned}
$$

Similarly, we have

$$
\begin{aligned}
\mathbf{I}_{52}= & -\frac{1}{2} \int_{0}^{t} \int_{\mathbb{T}^{3}} n^{\lambda} u^{\lambda} \cdot \nabla\left|u^{0}\right|^{2} d x d \tau=-\frac{1}{2} \int_{0}^{t} \int_{\mathbb{T}^{3}} \partial_{\tau}\left(n^{\lambda}-1\right)\left|u^{0}\right|^{2} d x d \tau \\
= & -\frac{1}{2} \int_{\mathbb{T}^{3}}\left(n^{\lambda}-1\right)\left|u^{0}\right|^{2} d x+\frac{1}{2} \int_{\mathbb{T}^{3}}\left(n_{0}^{\lambda}-1\right)\left|u_{0}^{0}\right|^{2} d x \\
& +\frac{1}{2} \int_{0}^{t} \int_{\mathbb{T}^{3}}\left(n^{\lambda}-1\right) \partial_{\tau}|u|^{2} d x d \tau
\end{aligned}
$$




$$
\begin{aligned}
\leq & -\frac{1}{2} \int_{\mathbb{T}^{3}}\left(n^{\lambda}-1\right)\left|u^{0}\right|^{2} d x+\frac{1}{2} \int_{\mathbb{T}^{3}}\left(n_{0}^{\lambda}-1\right)\left|u_{0}^{0}\right|^{2} d x \\
& +\frac{\lambda}{2} \int_{0}^{t} \int_{\mathbb{T}^{3}} \nabla \Psi^{\lambda} \cdot \nabla\left(\partial_{\tau}\left|u^{0}\right|^{2}\right) d x d \tau \\
\leq & -\frac{1}{2} \int_{\mathbb{T}^{3}}\left(n^{\lambda}-1\right)\left|u^{0}\right|^{2} d x+\frac{1}{2} \int_{\mathbb{T}^{3}}\left(n_{0}^{\lambda}-1\right)\left|u_{0}^{0}\right|^{2} d x+C \lambda .
\end{aligned}
$$

Then, we have

$$
\begin{aligned}
\mathbf{I}_{5} \leq & -\int_{0}^{t} \int_{\mathbb{T}^{3}} n^{\lambda} u^{\lambda} \cdot\left(\left(u^{0} \cdot \nabla\right) u^{0}\right) d x d \tau-\frac{1}{2} \int_{\mathbb{T}^{3}}\left(n^{\lambda}-1\right)\left|u^{0}\right|^{2} d x \\
& +\frac{1}{2} \int_{\mathbb{T}^{3}}\left(n_{0}^{\lambda}-1\right)\left|u_{0}^{0}\right|^{2} d x+C \lambda .
\end{aligned}
$$

Notice that the first term on the right hand side of the above inequality is canceled by the first term on the right hand side of (2.9). Meanwhile, we become aware of that the second and the third terms on the right hand side of the above inequality can be canceled by $\mathbf{I}_{2}$ and $\mathbf{I}_{3}$. Therefore, we have

$$
\mathbf{I}_{2}+\mathbf{I}_{3}+\mathbf{I}_{5} \leq-\int_{0}^{t} \int_{\mathbb{T}^{3}} n^{\lambda} u^{\lambda} \cdot\left(\left(u^{0} \cdot \nabla\right) u^{0}\right) d x d \tau+C \lambda .
$$

For the terms $\mathbf{I}_{6}$ and $\mathbf{I}_{7}$, it is easy to get

$$
\mathbf{I}_{6}+\mathbf{I}_{7} \leq C\left\|\nabla u^{0}\right\|_{L^{\infty}\left(0, T ; L^{\infty}\left(\mathbb{T}^{3}\right)\right)} \int_{0}^{t} \int_{\mathbb{T}^{3}}\left(\left|\nabla n^{\lambda}\right|^{2}+\left|\nabla \Psi^{\lambda}\right|^{2}\right) d x d \tau \leq C \int_{0}^{t} \mathcal{H}^{\lambda}(\tau) d \tau .
$$

Finally, we deal with $\mathbf{I}_{8}$. In view of Proposition 2, using the $L^{\infty}\left([0, T] ; L^{\gamma}\left(\mathbb{T}^{3}\right)\right)$ bound on $n^{\lambda}$, we deduced that $\sqrt{n^{\lambda}}$ is bounded in $L^{\infty}\left([0, T] ; L^{2 \gamma}\left(\mathbb{T}^{3}\right)\right)$ (then in $L^{\infty}\left([0, T] ; L^{2}\left(\mathbb{T}^{3}\right)\right)$ because $\left.\gamma>1\right)$ which combined with the $L^{2}\left([0, T] ; L^{2}\left(\mathbb{T}^{3}\right)\right)$ bound on $\sqrt{n^{\lambda}} \nabla u^{\lambda}$ gives

$$
\begin{aligned}
\mathbf{I}_{8} & =2 \mu \int_{0}^{t} \int_{\mathbb{T}^{3}} \sqrt{n^{\lambda}} \sqrt{n^{\lambda}} D\left(u^{\lambda}\right): \nabla u^{0} d x d \tau \leq C \mu \int_{0}^{t} \int_{\mathbb{T}^{3}} \sqrt{n^{\lambda}}\left|\sqrt{n^{\lambda}} D\left(u^{\lambda}\right)\right| d x d \tau \\
& \leq C \mu+2 \mu \int_{0}^{t} \int_{\mathbb{T}^{3}} n^{\lambda}\left|D\left(u^{\lambda}\right)\right|^{2} d x d \tau .
\end{aligned}
$$

Putting the estimates (2.8)-(2.11) into (2.4) and using the assumption $\mu=$ $O(\lambda)$, we obtain

$$
\mathcal{H}^{\lambda}(t) \leq C \int_{0}^{t} \mathcal{H}^{\lambda}(\tau) d \tau+C \lambda^{\min \left\{\frac{\gamma}{2}, 1\right\}}
$$

for all $t \in[0, T]$. With the help of Gronwall inequality, we get that

$$
\mathcal{H}^{\epsilon}(t) \leq C \lambda^{\min \left\{\frac{\gamma}{2}, 1\right\}}, t \in[0, T]
$$

which implies that the results (1.14) and (1.15) hold. Analogous to the proof of Lemma 1, we can easily prove the result (1.12), namely

$$
\left\|n^{\lambda}-1\right\|_{L^{\infty}\left([0, T] ; L^{\gamma}\left(\mathbb{T}^{3}\right)\right)}^{\gamma} \leq C \lambda^{\min \left\{\frac{\gamma^{2}}{4}, 1\right\}} .
$$


Using Lemma 1, the inequality (1.9), the inequality (2.7) and the Hölder inequality, we have that

$$
\begin{aligned}
\left\|\sqrt{n^{\lambda}} u^{\lambda}-u^{0}\right\|_{L^{2}\left(\mathbb{T}^{3}\right)}^{2} & \leq 2\left\|\sqrt{n^{\lambda}}\left(u^{\lambda}-u^{0}\right)\right\|_{L^{2}\left(\mathbb{T}^{3}\right)}^{2}+2\left\|\left(1-\sqrt{n^{\lambda}}\right) u^{0}\right\|_{L^{2}\left(\mathbb{T}^{3}\right)}^{2} \\
& \leq C \lambda^{\min \left\{\frac{\gamma}{2}, 1\right\}}+C\left\|1-n^{\lambda}\right\|_{L^{\gamma}\left(\mathbb{T}^{3}\right)}^{\gamma} \leq C \lambda^{\min \left\{\frac{\gamma^{2}}{4}, 1\right\}}
\end{aligned}
$$

for any $t \in[0, T]$. Therefore, we conclude that (1.13) holds. Using the Hölder inequality and the fact $1<\frac{2 \gamma}{\gamma+1}<\gamma$, we have

$$
\begin{aligned}
& \left\|n^{\lambda} u^{\lambda}-u^{0}\right\|_{L^{\frac{2 \gamma}{\gamma+1}}\left(\mathbb{T}^{3}\right)}^{2} \leq 2\left\|n^{\lambda}\left(u^{\lambda}-u^{0}\right)\right\|_{L^{\frac{2 \gamma}{\gamma+1}}\left(\mathbb{T}^{3}\right)}^{2}+2\left\|\left(n^{\lambda}-1\right) u^{0}\right\|_{L^{\frac{2 \gamma}{\gamma+1}}\left(\mathbb{T}^{3}\right)}^{2} \\
& \leq 2\left\|\sqrt{n^{\lambda}}\right\|_{L^{2 \gamma}\left(\mathbb{T}^{3}\right)}^{2}\left\|\sqrt{n^{\lambda}}\left(u^{\lambda}-u^{0}\right)\right\|_{L^{2}\left(\mathbb{T}^{3}\right)}^{2} \\
& +2\left\|n^{\lambda}-1\right\|_{L^{\gamma}\left(\mathbb{T}^{3}\right)}^{\gamma}\left\|u^{0}\right\|_{L^{\frac{2 \gamma}{\gamma-1}\left(\mathbb{T}^{3}\right)}}^{2} \leq C \lambda^{\min \left\{\frac{\gamma^{2}}{4}, 1\right\}}
\end{aligned}
$$

So we conclude that (1.16) holds. Thus the proof of Theorem 1 is finished.

\section{References}

[1] M. Bostan. The Vlasov-Maxwell system with strong initial magnetic field: guiding-center approximation. Multiscale Model. Simul, 6(3):1026-1058, 2007. https://doi.org/10.1137/070689383.

[2] Y. Brenier. Convergence of the Vlasov-Poisson system to the incompressible Euler equations. Commun. Part. Diff. Eqs., 25(3-4):737-754, 2000. https://doi.org/10.1080/03605300008821529.

[3] D. Bresch and B. Desjardins. On the existence of global weak solutions to the Navier-Stokes equations for viscous compressible and heat conducting fluids. J. Math. Pures Appl., 87(1):57-90, 2007. https://doi.org/10.1016/j.matpur.2006.11.001.

[4] D. Bresch, B. Desjardins and B. Ducomet. Quasi-neutral limit for a viscous capillary model of plasma. Ann. Inst. H. Poincaré Anal. Non Lianire, 22(1):19, 2005. https://doi.org/10.1016/j.anihpc.2004.02.001.

[5] S. Cordier and E. Grenier. Quasineutral limit of an Euler-Poisson system arising from plasma physics. Communications in Partial Differential Equations, 25(56):1099-1113, 2000. https://doi.org/10.1080/03605300008821542.

[6] D. Donatelli, E. Feireisl and A. Novotný. Scale analysis of a hydrodynamic model of plasma. M3AS. Math. Models Methods Appl. Sci, 25(2):371-394, 2015. https://doi.org/10.1142/S021820251550013X.

[7] D. Donatelli and P. Marcati. Analysis of oscillations and defect measures for the quasineutral limit in plasma physics. Arch. Rat. Mech. Anal., 206(1):159-188, 2012. https://doi.org/10.1007/s00205-012-0531-6.

[8] D. Donatelli and P. Marcati. The quasineutral limit for the Navier-StokesFourier-Poisson system. Springer Proceedings in Mathematics \& Statistics, 49:193-206, 2013. https://doi.org/10.1007/978-3-642-39007-4_9.

[9] D. Donatelli and P. Marcati. Quasi-neutral limit, dispersion and oscillations for Korteweg-type fluids. SIAM J. Math. Anal., 47(3):2265-2282, 2015. https://doi.org/10.1137/140987651. 
[10] J.E. Dunn and J. Serrin. On the thermomechanics of interstitial working. Arch. Rat. Mech. Anal., 88(2):95-133, 1985. https://doi.org/10.1007/BF00250907.

[11] I. Gasser, L. Hsiao, P. Markowich and S. Wang. Quasi-neutral limit of a nonlinear drift diffusion model for semiconductor models. J. Math. Anal. Appl., 268(1):184-199, 2002. https://doi.org/10.1006/jmaa.2001.7813.

[12] E. Grenier. Oscillations in quasineutral plasmas. Communications in Partial Differential Equations, 21(3-4):363-394, 1996. https://doi.org/10.1080/03605309608821189.

[13] Q.C. Ju, F.C. Li and S. Wang. Convergence of the Navier-Stokes-Poisson system to the incompressible Navier-Stokes equations. J. Math. Phys., 49(7):073515, 2008. https://doi.org/10.1063/1.2956495.

[14] A. Jüngel, C.K. Lin and K.C. Wu. An asymptotic limit of a Navier-Stokes system with capillary effects. Communications in Mathematical Physics, 329(2):725744, 2014. https://doi.org/10.1007/s00220-014-1961-9.

[15] T. Kato. Nonstationary flow of viscous and ideal fluids in $\mathbb{R}^{3}$. J. Funct. Anal, 9(3):296-305, 1972. https://doi.org/10.1016/0022-1236(72)90003-1.

[16] D.J. Korteweg. Sur la forme que prennent les équations du mouvement des fluides si l'on tient compte des forces capillaires causées par des variations de densité considé rables mais continues et sur la théorie de la capillarité dans l'hypothèse d'une variation continue de la densité. Archives Néerlandaises de Sciences Exactes et Naturelles, 6:1-24, 1901.

[17] F.C. Li. Quasineutral limit of the electro-diffusion model arising in electrohydrodynamics. J. Differential Equations, 246(9):3620-3641, 2009. https://doi.org/10.1016/j.jde.2009.01.027.

[18] F.C. Li. Quasineutral limit of the viscous quantum hydrodynamic model for semiconductors. J. Math. Anal. Appl., 352(2):620-628, 2009. https://doi.org/10.1016/j.jmaa.2008.11.011.

[19] H.L. Li and C.K. Lin. Zero Debye length asymptotic of the quantum hydrodynamic model for semiconductors. Comm. Math. Phys., 256(1):195-212, 2005. https://doi.org/10.1007/s00220-005-1316-7.

[20] Y.P. Li and W. A Yong. Quasi-neutral limit in a 3D compressible Navier-StokesPoisson-Korteweg model. IMA Journal of Applied Mathematics, 80(3):712-727, 2015. https://doi.org/10.1093/imamat/hxu008.

[21] P. Markowich, C.A. Ringhofer and C.A. Schmeiser. Semiconductor Equations. Springer-Verlag, 1955.

[22] N. Masmoudi. From Vlasov-Poisson system to the incompressible Euler system. Communications in Partial Differential Equations, 26(3):1913-1928, 2001. https://doi.org/10.1081/PDE-100107463.

[23] Y.J. Peng and S. Wang. Rigorous derivation of incompressible e-MHD equations from compressible Euler-Maxwell equation. SIAM J. Math. Anal., 40(2):540565, 2008. https://doi.org/10.1137/070686056.

[24] Y.J. Peng, Y.G.Wang and W.A. Yong. Quasi-neutral limit of the non-isentropic Euler-Poisson system. Proc. Roy. Soc. Edinburgh Sect. A, 136(5):1013-1026, 2006. https://doi.org/10.1017/S0308210500004856.

[25] M. Slemrod and N. Sternberg. Quasi-neutral limit for Euler-Poisson system. J. Nonlinear Sci., 11(3):193-209, 2001. https://doi.org/10.1007/s00332-001-00049 . 
[26] S. Wang. Quasineutral limit of Euler-Poisson system with and without viscosity. Communications in Partial Differential Equations, 29(3-4):419-456, 2005. https://doi.org/10.1081/PDE-120030403.

[27] S. Wang and S. Jiang. The convergence of the Navier-Stokes-Poisson system to the incompressible Euler equations. Comm. Partial. Differ. Equ., 31(4):571-591, 2006. https://doi.org/10.1080/03605300500361487.

[28] S. Wang, Z.P. Xin and P. Markowich. Quasi-neutral limit of the drift-diffusion models for semiconductors: the case of general signchanging doping profile. SIAM J. Math. Anal., 37(6):1854-1889, 2006. https://doi.org/10.1137/S0036141004440010.

[29] J.W. Yang and Q.C. Ju. Convergence of the quantum Navier-StokesPoisson equations to the incompressible Euler equations for general initial data. Nonlinear Anal. Real World Appl., 23:148-159, 2015. https://doi.org/10.1016/j.nonrwa.2014.12.003.

[30] J.W. Yang, Z.Y. Wang and F.X. Ding. Existence of global weak solutions for a 3D Navier-Stokes-Poisson-Korteweg equations. Applicable Analysis, 2017. https://doi.org/10.1080/00036811.2016.1276172. 